\title{
Abnormal expression and function of the E-cadherin-catenin complex in gastric carcinoma cell lines
}

A U Jawhari, M Noda, M J G Farthing and M Pignatelli

Correction to: British Journal of Cancer (1999) 80, 322-330. doi:10.1038/ sj.bjc. 6690358

It has been brought to our attention that there is a possible error in Figure 5 of the above paper whereby the western blot panel for beta-catenin representing the two cell lines MKN45 and MKN7 cells is identical. On inspection we agree with this observation, which is clearly an error on our part and which was also missed during the peer review process. Unfortunately we are unable to access the original gels and it is therefore not possible to offer the journal a full corrigendum, but nevertheless we feel it is important to acknowledge the error and formally put it on the record.

Figure 5 in this paper represents our study of the linkage between E-cadherin and beta-catenin in these cell lines. Previous (Shibamoto et al, 1994) and subsequent publications (Ono et al, 2004; Atsumi et al, 2007) looking at the E-cadherin-catenin complex in these cell lines have shown concordant results with the conclusions of our paper, where we show that despite the similar expression and interaction of the E-cadherin and betacatenin in both cell lines, cell to cell adhesion characteristics are different. Other authors have proposed this to be due to other factors influencing cellcell adhesion, such as differences in tyrosine phosphorylation levels of these proteins. Our paper is purely descriptive and our findings are supported by other publications in the field. We therefore conclude that this error in Figure 5 does not alter interpretation of the findings of our paper.
We have scrutinised the other data and images in the paper and can confidently confirm that there are no further errors or inaccuracies.

This note has been approved by all the authors below:

Current addresses: Aida U Jawhari, Queens Medical Centre, Nottingham, UK

Michael JG Farthing, Vice-Chancellor, Sussex House, University of Sussex, Brighton, UK

Massimo Pignatelli, Nazarbayev University Astana, Kazakhstan

\section{REFERENCES}

Atsumi T, Kato K, Uno K, Iijima K, Koike T, Imatani A, Ohara S, Shimosegawa T (2007) Pathophysiological role of the activation of p38 mitogen-activated protein kinases in poorly differentiated gastric cancer. Pathol Int 57(10): 635-644.

Ono H, Takeuchi Y, Ukegawa J, Kusayanagi S, Mitamura K, Imawari M (2004) Increased tyrosine kinase activities may lead to the phenotypic differences of gastric cancer cells. Anticancer Res 24(2B): 699-705.

Shibamoto S, Hayakawa M, Takeuchi K, Hori T, Oku N, Miyazawa K, Kitamura N, Takeichi M, Ito F (1994) Tyrosine phosphorylation of beta-catenin and plakoglobin enhanced by hepatocyte growth factor and epidermal growth factor in human carcinoma cell lines. Cell Adhes Commun 1(4): 295-305.

\section{Estimating the asbestos-related lung cancer burden from mesothelioma mortality}

V McCormack, J Peto, G Byrnes, K Straif and P Boffetta

Correction to: British Journal of Cancer (2012) 106, 575-584. doi:10.1038/ bjc. 2011.563

Following the publication of this paper, an omission was recognised. It is corrected below.

\section{CONFLICT OF INTEREST}

PB has acted as an expert witness in a trial involving asbestos exposure in the manufacture of synthetic polymers and risk of mesothelioma. VMcC, JP, GB and KS declare no conflicts of interest.

\section{Farnesoid X receptor, overexpressed in pancreatic cancer with lymph node metastasis promotes cell migration and invasion}

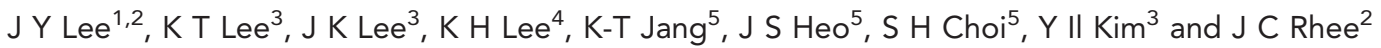

${ }^{1}$ Department of Health Science and Technology, Graduate School, SAlHST, Sungkyunkwan University, Seoul, Korea; ${ }^{2}$ Samsung Biomedical Research Institute, Seoul, Korea; ${ }^{3}$ Department of Medicine, Samsung Medical Center, 50 Irwon-dong, Gangnam-gu, Sungkyunkwan University School of Medicine, Seoul 135-710, Korea; ${ }^{4}$ Department of Pathology, Sungkyunkwan University School of Medicine, Seoul, Korea and ${ }^{5}$ Department of Surgery, Samsung Medical Center, Sungkyunkwan University School of Medicine, Seoul, Korea

Correction to: British Journal of Cancer (2011) 104, 1027-1037. doi:10.1038/ bjc.2011.37

Published online 31 July 2014
Owing to an error on the author's part the affiliation given for the first author, Ms JY Lee, was incomplete. The full and correct affiliations are now given above. 